\title{
APLICACIÓN DE SERIES DE TIEMPO EN LA REALIZACIÓN DE PRONÓSTICOS DE PRODUCCIÓN
}

Erik Giovany Montes Páez ${ }^{*}$. Fernando Enrique Calvete González², Carlos Alfonso Mantilla Duarte ${ }^{3}$

* A quien debe dirigirse la correspondencia.

\begin{abstract}
RESUMEN
La generación de pronósticos de producción es una actividad cotidiana del ingeniero de petróleos, que se ha venido desarrollando mediante la implementación de herramientas computacionales basadas en los modelos de curvas de declinación y curvas tipo. Estos modelos presentan falencias en la exactitud de los pronósticos, básicamente por dos razones: primero, tienen como gran condición el hecho de que los pozos se encuentren operando en estado pseudoestable y, segundo, ajustan el comportamiento de la producción a una línea de tendencia, la cual es extrapolada en el tiempo para obtener los pronósticos. En esta investigación, como una alternativa a estos modelos, se ha propuesto el uso de series de tiempo en la generación de pronósticos, pues estas incluyen tanto la tendencia como los componentes cíclicos y estacionales de los datos de producción. Se comparó el error absoluto existente entre los datos reales y los pronósticos obtenidos por metodologías convencionales y la aplicación de modelos de series de tiempo. El uso de estas series permitió obtener un mejor ajuste histórico de los datos, evidenciar que pueden presentarse otras tendencias en la declinación (como la cúbica, por ejemplo) y aumentar la precisión de los pronósticos generados.
\end{abstract}

Palabras clave: Producción, Pronósticos, Series de tiempo, Modelo ARIMA.

TIME SERIES APPLIED IN PRODUCTION FORECASTING

\section{ABSTRACT}

Production forecasting is a daily activity in Petroleum Engineering, developed by commercial software based in Decline Curve Analysis and Type Curves. These models have failures in two aspects: the first one, models are conditioned to wells producing under pseudosteady state; second one, the production data is fitted to a tendential line, that is extrapolated in the time for get the forecasting. In this investigation, as an alternative to those models, a time series application is proposed, because time series include tendencial, cyclic and stational components of production data. The error between the actual data was compared to the forecasts obtained by conventional methods and results of the time series model. This application allowed to obtain a better history matching of data, evidence that other trends may be in decline (cubic, for example) and increase the accuracy of forecasts generated.

Keywords: Production, Forecasting, Time Series, ARIMA Model.

1. Grupo de investigación modelamiento de procesos de hidrocarburos - GMPH, Escuela de Ingeniería de Petróleos, Universidad Industrial de Santander, UIS, Carrera 27calle 9, Bucaramanga, Colombia. erik.montes@, correo.uis.edu.co

2. Grupo de investigación modelamiento de procesos de hidrocarburos - GMPH, Escuela de Ingeniería de Petróleos, Universidad Industrial de Santander, UIS, Carrera 27calle 9, Bucaramanga, Colombia. fcalvete@uis. edu.co

3. Universidad de Granada, UGR, Granada, España. cmanduar@correo.ugr.es 


\section{INTRODUCCIÓN}

En la actualidad, las técnicas empleadas en la generación de pronósticos de producción se basan en los modelos de "Curvas de declinación" (Arps, 1945) y de "Curvas Tipo" (Fetkovich, 1980; Palacio \& Blasingame, 1993), cuyo uso se refleja en la aparición de softwares comerciales para el seguimiento y predicción de la producción de pozos.

En estos programas se plantean técnicas de análisis integrado de datos, cuyo objetivo es la obtención de pronósticos de producción, a partir de la generación y extrapolación de líneas de tendencia. Sin embargo, al analizar estas técnicas se pueden identificar algunos puntos que se consideran como poco ventajosos:

- Requieren que los pozos se encuentren produciendo en estado pseudoestable, es decir, que la presión de fondo fluyente de los pozos sea constante. Esta condición difícilmente se satisface debido a que diversas situaciones (acumulación de gas en el anular del pozo, cambios en la eficiencia de los sistemas de levantamiento artificial, daño a la formación, reducción de la capacidad de las tuberías, etc.) generan variaciones en las condiciones de presión del pozo.

- Asumen que las variables tienen un comportamiento paramétrico, es decir, que se rigen por tendencias normales que pueden ser extrapoladas. En realidad, pueden ocurrir varias situaciones. Por ejemplo, que un pozo tenga varias tendencias de producción a lo largo de su vida productiva, según se produjeron los fenómenos anteriormente citados; que los datos presenten fluctuaciones alrededor de la línea de tendencia; o que la extrapolación de dicha tendencia no permita obtener un buen ajuste de la predicción.

- Presentan fallas cuando no se posee la información completa, suficiente y necesaria para aplicarlos. Siendo esta una fuerte limitante en campo, donde no siempre se cuenta con datos actualizados en áreas como fluidos, petrofísica y análisis de presiones.

- No involucran la probabilidad de la ocurrencia de ciertas circunstancias (tales como fallas en los sistemas de producción, intervenciones a los pozos, cambio de las condiciones operacionales) respuestas del sistema de producción ante las acciones tomadas.
Teniendo en cuenta estas razones, se pretende buscar el planteamiento de nuevas metodologías para el seguimiento del desempeño de un yacimiento y la predicción de su comportamiento futuro, a partir de la aplicación de series de tiempo.

Estas series se basan en la suposición de que los datos actuales se pueden calcular a partir del análisis de los datos anteriores, pero no simplemente considerando la tendencia de los mismos, sino que incluyen las fluctuaciones propias de las variables a lo largo del tiempo, representadas en componentes cíclicas y estacionales.

El objetivo consiste en evaluar qué tanto pueden mejorarse las predicciones realizadas por las series de tiempo con respecto a los resultados obtenidos con los modelos tradicionales.

\section{CURVAS DE DECLINACIÓN}

Desde la primera mitad del siglo XX, en la industria petrolera se han empleado las curvas de declinación como la principal herramienta para la obtención de pronósticos de producción. Aun cuando algunas de sus consideraciones difícilmente se satisfacen en la práctica.

Una curva de declinación tiene dos parámetros que la describen. El primero, la tasa de declinación $(D)$, que refleja el ritmo al cual disminuye la producción de un pozo en términos de porcentaje diario, mensual o anual, y que se calcula mediante la ecuación 1. Donde $\mathrm{q}_{1} \mathrm{y} \mathrm{q}_{2}$ son, respectivamente, las tasas de producción del pozo medidas en los tiempos $\mathrm{t}_{1} \mathrm{y} \mathrm{t}_{2}$.

$$
D=\frac{\left(q_{1}-q_{2}\right)}{q_{1}\left(t_{2}-t_{1}\right)}
$$

Y el segundo, el exponente de declinación (n), que se ve reflejado en la tendencia de la curva de declinación (ver Figura 1), donde se observa que esta puede variar desde una situación en la que la declinación sea muy severa (declinación exponencial, con un exponente $\mathrm{n}=0$ ), hasta una condición de reducción lenta de la tasa de producción (declinación armónica, con un exponente $\mathrm{n}=1$ ), pasando por casos intermedios (declinación hiperbólica, $0<\mathrm{n}<1)$. 


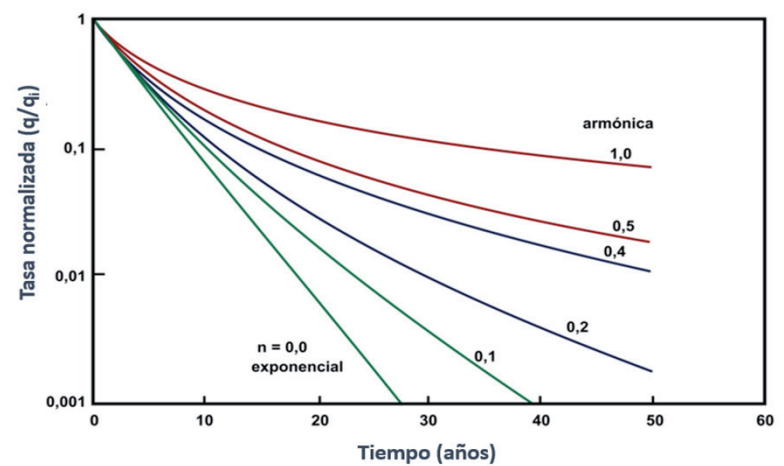

Figura 1. Modelos de declinación (Poston, 2008).

El exponente de declinación puede determinarse de diferentes maneras, bien sea mediante la generación de gráficas (Mannon, 1964; Aderemi, 2008; Poston, 2008), la identificación de tendencias (Fetkovich et al, 1994; Campbell, 1989) o con el uso de curvas tipo (Fetkovich, 1980; Palacio \& Blasingame, 1993).

Una vez se ha determinado el exponente declinación, pueden emplearse las ecuaciones que se presentan de manera resumida en la tabla 1, para el cálculo de las tasas de producción futuras, el petróleo producido acumulado en un determinado instante de la vida del pozo o el tiempo restante para alcanzar determinada tasa de producción. Dichas expresiones se obtienen del modelo propuesto por Arps, que se presenta en la ecuación 2.

$$
\frac{D}{D_{i}}=\left(\frac{q}{q_{i}}\right)^{n}
$$

Los pronósticos obtenidos con esta técnica son simples extrapolaciones de una línea de tendencia generada a partir de la data histórica de producción de un pozo, y no tienen en cuenta las fluctuaciones naturales asociadas al proceso de extracción de petróleo del subsuelo, por lo cual, suelen estar asociados a errores considerables.

El impacto de estos errores es enorme, pues a partir de los pronósticos de producción se toman muchas de las decisiones de inversión en los campos de producción, se cuantifican los gastos operacionales, se generan compromisos de producción, se generan contratos de venta de crudo, entre muchos otros aspectos relacionados con el gerenciamiento de un activo petrolero.

Tabla 1. Ecuaciones características para los modelos de declinación.

$\begin{array}{cccc}\text { Declinación } & \text { Exponencial } & \text { Hiperbólica } & \text { Armónica } \\ \text { Exponente de declinación } & n=0 & 0<n<1 & n=1 \\ \text { Tasa de declinación } & D=\frac{\left(q_{1}-q_{2}\right)}{q_{1} \Delta t} & D=\frac{D_{i}}{1+n D_{i} t} & D=\frac{D_{i} q}{q_{i}} \\ \text { Tasa de producción } & q=q_{i} e^{-D t} & q=q_{i}\left(1+n D_{i} t\right)^{-1 / n} & q=\frac{q_{i}}{1+D_{i} t} \\ \text { Producción acumulada } & N_{P}=\frac{q_{i}-q}{D} & N_{P}=\frac{q_{i}{ }^{n}\left(q_{i}{ }^{1-n}-q^{1-n}\right)}{D_{i}(1-n)} & N_{P}=\frac{q_{i}}{D_{i}} \operatorname{In}\left(\frac{q_{i}}{q}\right) \\ \text { Tiempo de abandono } & t_{a}=\frac{-\operatorname{In}\left(q_{i} / q_{a}\right)}{\operatorname{In}(1-D)} & t_{a}=\frac{1}{n D_{i}}\left[\left(\frac{q_{i}}{q}\right)^{n}-1\right] & t_{a}=\frac{1}{D_{i}}\left(\frac{q_{i}}{q_{a}}-1\right)\end{array}$

\section{SERIES DE TIEMPO}

Una serie de tiempo (o serie cronológica) es "una secuencia de datos empíricos ordenados en función del tiempo; esto es, que puedan graficarse contra el tiempo" (García, 2010), por lo que es aplicable a un conjunto de datos registrados periódicamente, ya sea un registro diario, semanal, semestral, mensual o anual, entre otros; siendo algunos ejemplos prácticos las ventas anuales totales de almacenes, el valor trimestral del producto interno bruto o, en este caso, la producción de uno o varios pozos de petróleo.
Para describir una serie de tiempo se deben identificar los siguientes componentes:

a) Tendencia o componente tendencial: es el resultado de factores que afectan a largo plazo a los datos, los cuales generan un patrón gradual y consistente de las variaciones de la misma serie. En el caso de un pozo, la tendencia de los datos de producción normalmente es la declinación, debida a fenómenos como la despresurización del yacimiento, el agotamiento de los fluidos presentes en él, o la ocurrencia del daño a la formación. De acuerdo con las metodologías 
tradicionales, la tendencia puede ser exponencial, hiperbólica o armónica, pero en el análisis de datos pueden considerarse otras tendencias, como la lineal, la cuadrática o la cúbica.

b) Componentes cíclicas y estacionales: tanto las variaciones cíclicas como las estacionales, pueden entenderse como el movimiento ondulante de los datos por encima y por debajo de la línea de tendencia. Pero la diferencia entre uno y otro tiene que ver con el rango de datos que se analizan. En el caso de las componentes cíclicas, se refiere a procesos que ocurren de manera más espaciada dentro del intervalo de observación, mientras que las componentes estacionales ocurren con menor separación en el tiempo.

Por ejemplo, al hablar de la operación de un pozo de petróleo, un componente estacional puede referirse a las variaciones de la tasa de producción que ocurren cada día (en las horas más calurosas se incrementa la tasa, mientras que en las noches frescas se reduce); mientras que una componente cíclica puede estar dada por procesos de reducción paulatina del caudal generados por el daño a la formación, y un posterior aumento en la tasa, luego de realizar estimulaciones periódicas al pozo.

c) Componente irregular: que se relaciona con datos muy desviados de la tendencia, como ocurre cuando se presenta una parada de producción en un pozo. En este caso, es necesario tomar decisiones en cuanto al manejo que se le da a estos valores anormales, pues incluirlos en los cálculos añadiría una gran cantidad de ruido a la descripción de los mismos. Por esta razón, suele estimarse un valor, para reemplazar el dato anormal.

Los modelos de serie de tiempo pueden clasificarse en cuatro grandes categorías, que pueden identificarse mediante la aplicación de la Metodología Box-Jenkins (Jiménez et al, 2006), mediante la cual se calculan las funciones de autocorrelación simple (F.A.S.) y de autocorrelación parcial (F.A.P.), cuyas formas típicas se presentan en la Figura 2.

\subsection{MODELOS AUTORREGRESIVOS}

Una de las características que puede tener una serie de tiempo es que los valores de la variable de estudio (en este caso, la producción) dependan de sus valores anteriores. En términos matemáticos, un modelo autorregresivo (AR) calcula el valor de la variable $y$ en el instante $t$ mediante la ecuación 3 .

$$
y_{t}=a+b y_{t-1}+\epsilon_{t}
$$

Donde $a$ y $b$ son constantes propias de cada serie de tiempo y $\varepsilon_{t}$ es el ruido blanco. En este caso, se está haciendo alusión a un modelo AR de orden 1, pues al calcular el valor de la variable de interés $(y)$ en el instante $t$, se está tomando un dato anterior al que se desea predecir (en este caso $y_{t-1}$ ). De manera análoga existen expresiones para modelos de mayor orden, incluyendo mayor cantidad de constantes de ajuste.

Adicionalmente, la determinación del orden del modelo (esto es, de la cantidad de valores previos que deben emplearse para el cálculo de un valor futuro) se realiza mediante la determinación del "Valor P" (Schwarz, 1978; Sterne, 2001) que se considera como una medida de qué tan probable es que las predicciones de un modelo se acerquen a la realidad. Este parámetro oscila entre cero y uno, y mientras más alto sea el "Valor P", mayor probabilidad existe de que el modelo propuesto describa acertadamente los datos.

\subsection{MODELOS DE MEDIA MÓVIL}

En otros casos, los datos de una serie de tiempo dependen del ruido blanco que se encuentre asociado a cada uno de las observaciones que constituyen la serie. En este caso, no sólo se incluye un término que resume los ruidos de la totalidad de la serie $\left(\varepsilon_{\mathrm{t}}\right)$ sino que en cada observación $\left(\mathrm{y}_{\mathrm{i}}\right)$ se deberá agregar el ruido correspondiente $\left(\varepsilon_{\mathrm{i}}\right)$. La forma general de un modelo de media móvil se presenta en la ecuación 4.

$$
y_{t}=a+\epsilon_{t}+\theta_{1} \epsilon_{t-1}+\ldots+\theta_{q} \epsilon_{t-q}
$$

Donde $a, \theta_{l}, \theta_{2}, \theta_{q}$, son parámetros que deben determinarse para el modelo, y $q$ es orden del modelo, es decir, el número de valores pasados que afectan el cálculo del valor actual.

Si el modelo de media móvil es de orden 1, su expresión sería la presentada en la ecuación 5.

$$
y_{t}=a+\epsilon_{t}+\theta_{1} \epsilon_{t-1}
$$

\subsection{MODELOS "ARMA" Y "ARIMA"}

El análisis de los modelos AR y de los modelos MA resulta sencillo, pues simplemente se está considerando una componente de la serie de tiempo (la tendencia de los últimos datos o el error de los últimos datos, respectivamente), pero es poco usual que esto ocurra, pues en la mayoría de los casos coexisten ambas influencias. 


\begin{tabular}{|c|c|c|}
\hline MODELO & $\begin{array}{c}\text { Función de autocorrelación } \\
\text { simple (FAS) }\end{array}$ & $\begin{array}{c}\text { Función de autocorrelación } \\
\text { parcial (FAP) }\end{array}$ \\
\hline $\begin{array}{l}\text { Autorregresivo } \\
\text { (AR) }\end{array}$ & & \\
\hline $\begin{array}{c}\text { De media } \\
\text { móvil (MA) }\end{array}$ & & \\
\hline ARMA & & \\
\hline ARIMA & & \\
\hline
\end{tabular}

Figura 2. Curvas típicas de las funciones de autocorrelación para diferentes modelos de serie de tiempo (Schwarz, 1978).

En estas situaciones, debe recurrirse a los modelos autorregresivos de media móvil, más conocidos como modelos ARMA(p, $\mathrm{q})$, donde $p$ y $q$ representan, respetivamente, el orden de autorregresión y el orden de media móvil. La expresión general de un modelo $\operatorname{ARMA}(1,1)$ sería la presentada en la ecuación 6.

$$
y_{t}=a+b y_{t-1}+\epsilon_{t}+\theta \epsilon_{t-1}
$$

Los modelos presentados (AR, MA y ARMA) tienen una característica común: son usados para representar procesos estocásticos estacionarios, es decir, que las variables están ordenadas cronológicamente y que la distribución de probabilidad de cada observación permanece constante en el tiempo. Pero, en algunos casos no se cumple con esta condición de una serie cronológica estacionaria, y la realización de pronósticos utilizando modelos como los ya vistos tendrá un gran error asociado, razón por lo cual Box y Jenkins plantearon los modelos ARIMA, que además de contener un componente autorregresivo y uno de media móvil, incluyen un componente de integración (de allí su nombre, como sigla en inglés de "autoregressive integrated moving average").
Este término de integración pretende, de forma numérica, llevar una serie no estacionaria a tener un comportamiento estacionario. En cuyo caso, los modelos numéricos resultan más complejos, como se presenta en la ecuación 7.

$$
\begin{aligned}
y_{t}= & a+b_{1} y_{t-1}+\ldots+b_{q} y_{t-q}+\epsilon_{t} \\
& +\theta_{1} \epsilon_{t-1}+\ldots+\theta_{q} \epsilon_{t-q}
\end{aligned}
$$

Precisamente, por la gran cantidad de datos a analizar, la complejidad de los cálculos y la enorme cantidad de opciones en cuanto a órdenes de los modelos se refiere, es que este tipo de análisis estadísticos deben realizarse por personal con gran experiencia en el manejo de datos y con la ayuda de software especializado.

\section{METODOLOGÍA DE ANÁLISIS DE PRODUCCIÓN MEDIANTE SERIES DE TIEMPO}

Con el objetivo de evaluar la precisión de los pronósticos que se obtienen con el uso de series cronológicas, se realizó el análisis de la data de producción de tres 
pozos ubicados en la cuenca de los Llanos Orientales, usando la metodología planteada y comparando dichos resultados con los que se obtuvieron al emplear cuatro programas comerciales:

- Oilfield Manager (OFM) de la compañía Schlumberger.

- ValNav de Energy Navigator.

- Topaze de Kappa Engineering Group.

- Forecasting de Ryder Scott.

La metodología planteada para predecir la producción mediante series de tiempo se resume en cuatro pasos: manejo de datos irregulares, ajuste tendencial histórico, obtención del modelo de serie de tiempo y generación de pronósticos.

a) Manejo de datos irregulares: Incluir los valores de cero producción en el análisis de los datos no resulta conveniente porque, suponiendo que hubiese ocurrido una falla que impidió producir un cierto volumen de petróleo en un día específico, de no haberse producido dicha falla, la producción del pozo no habría sido cero. Es decir, que incluir un valor de "cero producción" en el análisis de los datos, implicaría que se está suponiendo que ese valor (cero) corresponde al comportamiento natural del pozo, lo cual evidentemente es incorrecto.

Por tanto, se recomienda asignar algún valor a la fecha en la que aparecen los datos irregulares. En este caso, se opta por la utilización de la "media móvil", técnica en la cual se reemplaza el valor faltante por el promedio de los datos de los días previos a la fecha en cuestión.

En este caso, se usaron de los datos de la semana previa al evento, de acuerdo con la ecuación 8 .

$$
q_{i}=\frac{q_{i-7}+q_{i-6}+\ldots+q_{i-2}+q_{i-1}}{7}
$$

Esta suposición se basa en un principio estadístico, según el cual, para un grupo de datos con distribución normal, la esperanza equivale a la media.

b) Ajuste tendencial histórico: Los análisis de regresión son procesos estadísticos que buscan establecer la relación existente entre variables. Si se tienen mediciones de cierta variable (por ejemplo, la producción de un pozo) a lo largo del tiempo, se trataría de establecer, mediante una ecuación, el comportamiento de dicha variable con respecto al tiempo.

En este caso, mediante la aplicación del método de mínimos cuadrados se procede a verificar el ajuste de la data de producción a diferentes tendencias: lineal, cuadrática, cúbica y exponencial.

En la tabla 2 se presenta un resumen de los criterios de información que permiten verificar el ajuste de los datos a las tendencias propuestas:

- El coeficiente de determinación (R2): Representa la relación existente entre las variaciones (desviaciones) explicadas por el modelo y las variaciones totales existentes. Es decir, un valor de R2 igual a uno quiere decir que todos los valores que presentan alguna variación con respecto a la tendencia están descritos por el modelo. Como criterio de decisión, se debe seleccionar aquel modelo que presente el valor de R2 más cercano a la unidad.

- El error cuadrático medio (MSE): Este criterio, como su nombre lo indica, mide el promedio de los errores de cada dato al cuadrado. Por lo tanto, mientras más pequeño sea el valor del MSE, mejor es el modelo estudiado.

- El criterio de información de Akaike (ACI): Es una medida de la calidad relativa del modelo que se está planteando para la descripción del fenómeno. Presenta una estimación de la información perdida cuando se utiliza el modelo planteado para describir los datos. Es decir, que mientras más pequeño sea el valor del ACI, menor información se está perdiendo y, por lo tanto, el modelo es más acertado.

- El criterio de información de Bayes (BCI): De manera análoga al ACI, este criterio permite identificar el mejor modelo. El BIC se relaciona con la desviación de los datos reales con respeto al modelo, incluyendo algunas correcciones bayesianas. La definición dice que "el BIC incluye la variación no explicada de la variable dependiente", es decir, que a medida que la descripción de la variable sea más pobre, mayor será el valor del BIC. En otras palabras, el mejor modelo es aquel que posea el menor valor del BIC.

Como se observa, en este caso el mejor ajuste se presentó para la tendencia cúbica, en desmedro del ajuste exponencial que se usa comúnmente.

En la Figura 3 se presentan las observaciones históricas de producción de uno de los pozos de estudio, junto con las curvas de tendencia propuestas. 
c) Obtención del modelo de serie de tiempo: En este punto deben determinarse dos aspectos muy importantes, el tipo de modelo y el orden del modelo.

El primer aspecto se relaciona con la identificación de cuál es la ecuación que permite describir con mayor precisión los datos históricos de producción, incluyendo su tendencia y sus componentes cíclicos y estacionarios. Para tal fin debe aplicarse la metodología BoxJenkins, con la se generan los gráficos de la "Función de autocorrelación simple" (F.A.S.) y la "Función de autocorrelación parcial" (F.A.P.) de los datos. En la Figura 4 se presentan las gráficas obtenidas numéricamente para los datos del pozo de estudio, verificando que puede emplearse un modelo ARIMA en el análisis posterior, pues las gráficas coinciden con lo propuesto en la figura 2 .

Seguidamente, mediante prueba y error, es necesario determinar el orden del modelo, es decir, qué cantidad de datos hacia atrás deben emplearse para calcular un nuevo valor en la serie cronológica.

Este análisis se realiza por medio del cálculo del "Valor P" que, como se mencionó anteriormente, se relaciona con el ajuste del modelo. Como se presenta en la tabla 3 , en este caso el modelo seleccionado es un modelo ARIMA(10,1,10).

Tabla 2. Coeficientes de ajuste de los cuatro modelos tendenciales para el pozo Montes 1.

\begin{tabular}{|ccccc|} 
Modelo & Lineal & Cuadrático & Cúbico & Exponencial \\
\hline $\mathbf{R}^{2}$ ajustado & 0,7085874 & 0,8463709 & 0,8585558 & 0,8558267 \\
\hline MSE & $4,197936 \times 10^{6}$ & $2,213099 \times 10^{6}$ & $2,037570 \times 10^{6}$ & $2,076884 \times 10^{6}$ \\
\hline AIC & 15,25050 & 14,61050 & 14,52807 & 14,54678 \\
\hline BIC & 15,52311 & 14,61441 & 14,53328 & 14,54938 \\
\hline Error absoluto (\%) & 12,50 & 7,38 & 6,35 & 6,60 \\
\hline
\end{tabular}

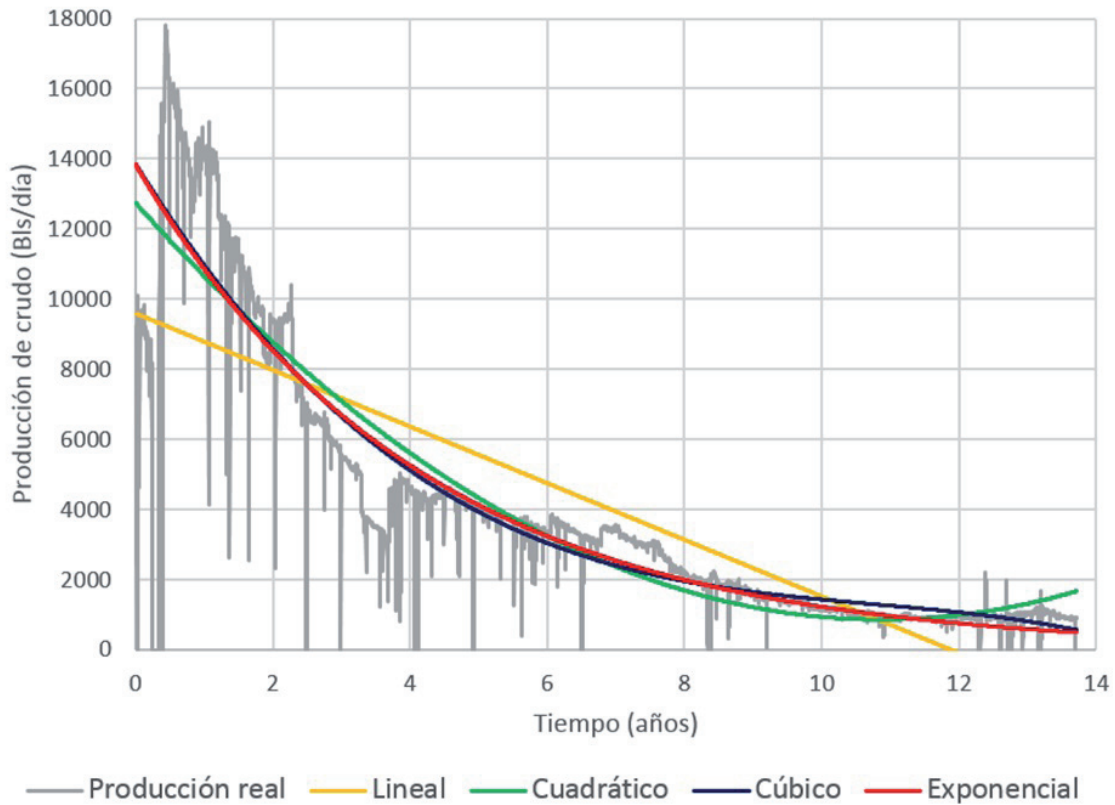

Figura 3. Ajuste histórico de la producción del pozo Montes 1. 

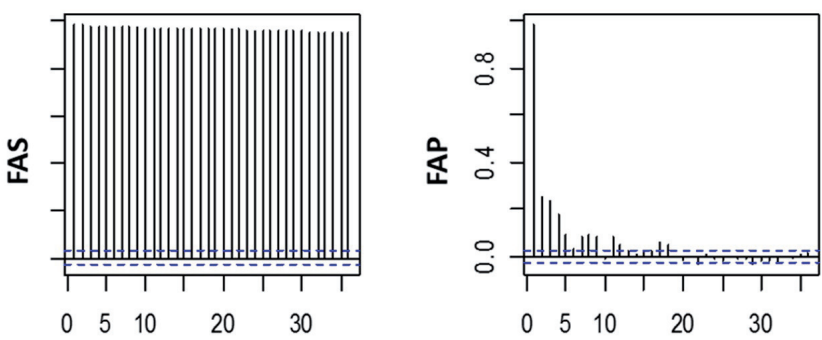

Figura 4. Funciones de autocorrelación simple (izquierda) y autocorrelación parcial para los datos del pozo Montes 1.

Tabla 3. "Valor P" para diferentes órdenes del Modelo ARIMA.

\begin{tabular}{ccccccc} 
Orden del Modelo & $\mathbf{1 , 1 , 1}$ & $\mathbf{2 , 1 , 2}$ & $\mathbf{3 , 1 , 3}$ & $\mathbf{4 , 1 , 4}$ & $\mathbf{5 , 1 , 5}$ & $\mathbf{6 , 1 , 6}$ \\
"Valor P" & $8,93 \times 10^{-10}$ & $5,07 \times 10^{-9}$ & $4,01 \times 10^{-9}$ & 0,01109 & 0,05985 & 0,4054 \\
Orden del Modelo & $\mathbf{7 , 1 , 7}$ & $\mathbf{8 , 1 , 8}$ & $\mathbf{9 , 1 , 9}$ & $\mathbf{1 0 , 1 , 1 0}$ & $\mathbf{1 1 , 1 , 1 1}$ & $\mathbf{1 2 , 1 , 1 2}$ \\
"Valor P" & 0,07178 & 0,9935 & 0,9943 & 0,9965 & 0,8080 & 0,6954 \\
\hline
\end{tabular}

d) Generación de pronósticos: Es la etapa final del proceso, donde se hacen los cálculos, a partir del modelo obtenido, para establecer los valores futuros.

Debido al orden del modelo, las componentes cíclica y estacionaria tienen un efecto sobre los pronósticos en el corto plazo (tal como puede apreciarse en la figura 5), pero este efecto se atenúa cuando se pretende continuar avanzando en el tiempo, llegando a obtener simplemente una línea de tendencia después de dos meses de pronóstico. En este caso, la producción fue pronosticada a partir del día 5006 de operación del pozo. Cabe resaltar que entre los días 5045 y 5170 se registraron paradas de producción que el modelo desarrollado no está en capacidad de predecir.

En la figura 6 se presenta una comparación de los resultados obtenidos para los cuatro software comerciales y los pronósticos generados con el modelo de serie de tiempo.

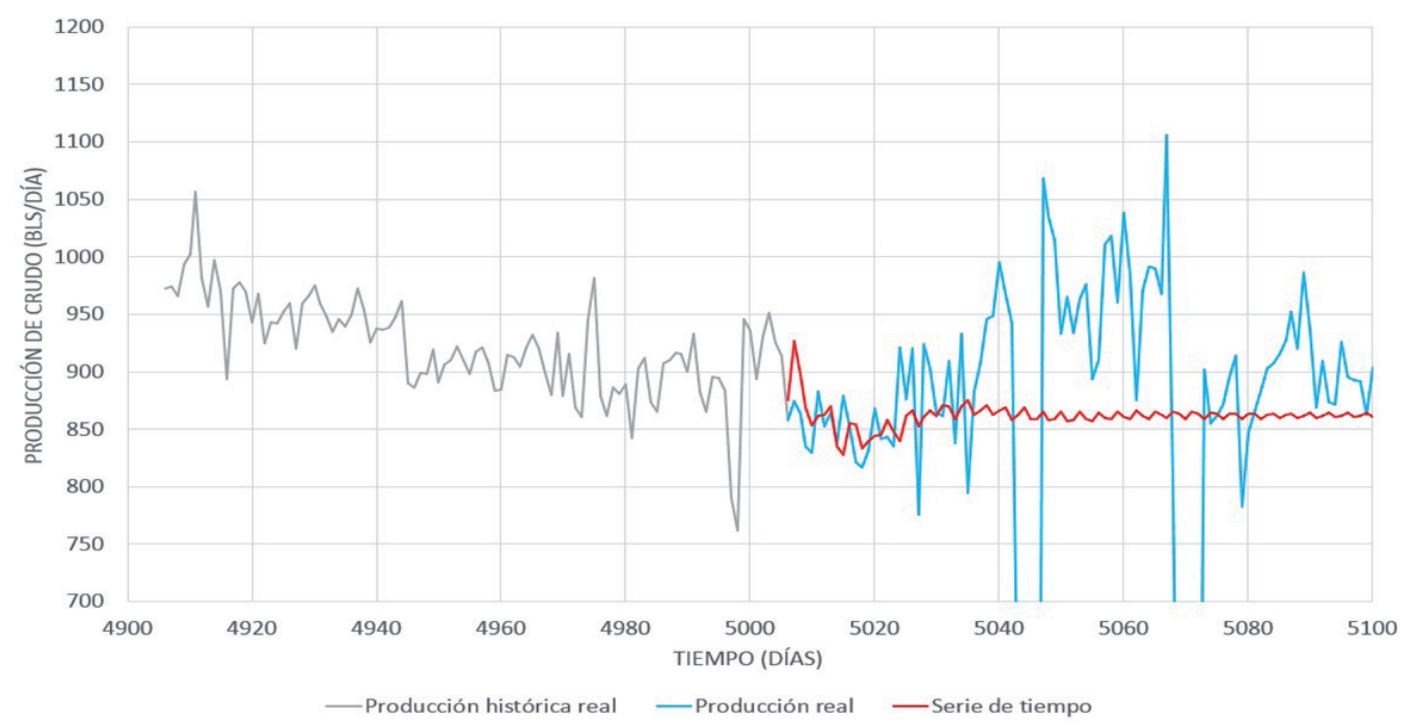

Figura 5. Pronóstico obtenido mediante el modelo de serie de tiempo. 


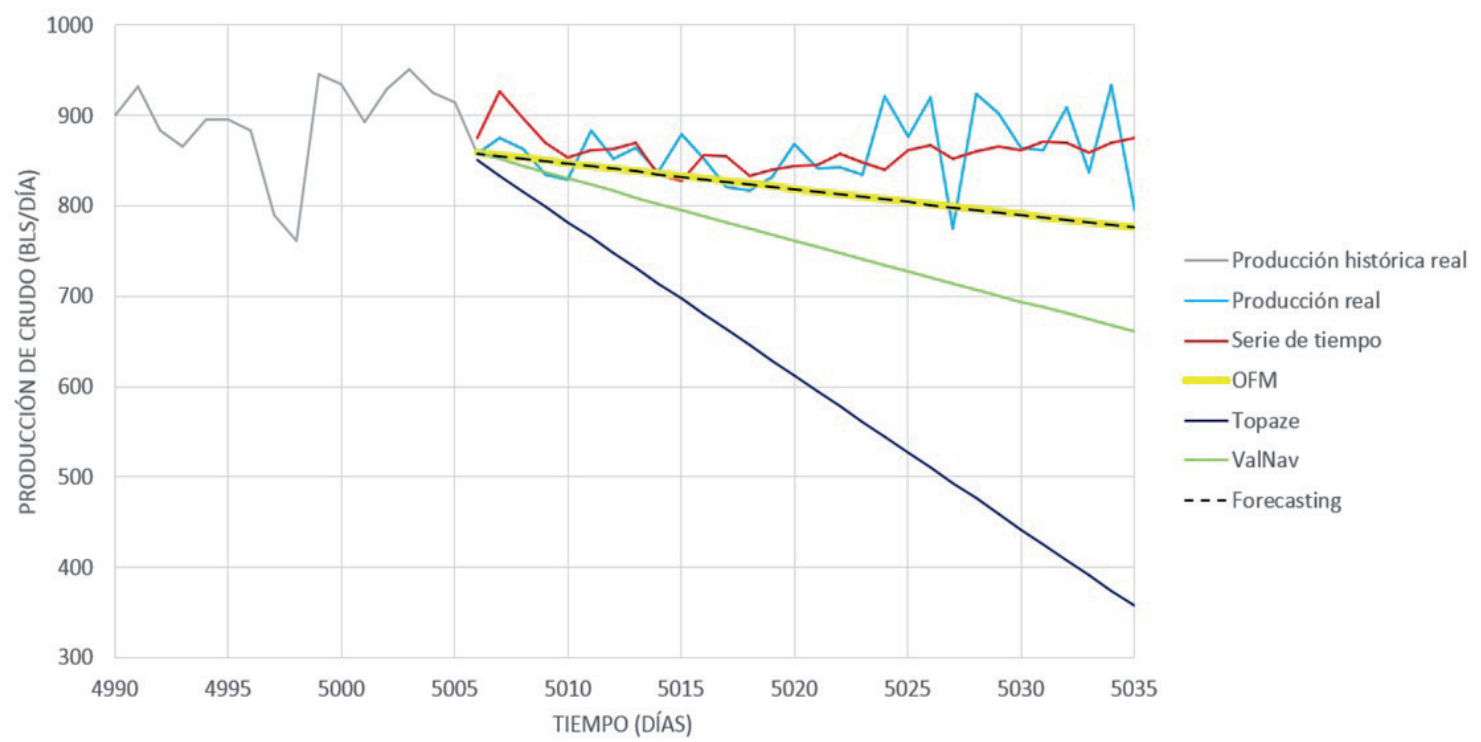

Figura 6. Comparación de pronósticos

\section{RESULTADOS}

En la tabla 4 se presenta un resumen de los errores obtenidos luego de generar pronósticos de producción por cinco métodos para un lapso de un mes para tres pozos diferentes. Dado que algunos programas emplean algoritmos similares, los resultados que se obtienen a partir de ellos pueden ser muy semejantes.

Tabla 4. Porcentajes de error para los diferentes métodos empleados, para un periodo de un mes.

\begin{tabular}{cccc} 
Técnica empleada & \multicolumn{3}{c}{ Error absoluto (\%) } \\
\hline POZO & Montes 1 & Montes 2 & Montes 3 \\
\hline OFM & 5,34 & 7,18 & 1,75 \\
\hline Topaze & 28,58 & 15,51 & 2,05 \\
\hline ValNav & 11,64 & 9,41 & 2,14 \\
\hline Forecast & 5,39 & 8,31 & 1,71 \\
\hline Serie de tiempo & 3,51 & 3,30 & 2,14 \\
\hline
\end{tabular}

En este caso, los datos generados por los programas Oilfield Manager y Forecasting, tienen valores muy cercanos, razón por la cual gráficamente resultan líneas superpuestas y los porcentajes de error de ambos programas son prácticamente iguales.

Como se puede apreciar, la implementación de la metodología de series cronológicas permite reducir significativamente el error de la predicción, gracias a que estos modelos consideran tanto la tendencia de producción del pozo (lo cual también está implícito en las herramientas software) como las oscilaciones que se presentan naturalmente en el pozo.

El análisis se repitió para un periodo más extenso (un año), obteniendo los resultados que se presentan en la tabla 5. La precisión de los resultados es muy deficiente, debido a que en un periodo tan extenso como el de un año las pequeñas desviaciones iniciales de los modelos se tornan en grandes márgenes de error.

Tabla 5. Porcentajes de error para los diferentes métodos empleados, para un periodo de un año.

\begin{tabular}{cccc} 
Técnica empleada & \multicolumn{3}{c}{ Error absoluto (\%) } \\
\hline POZO & Montes 1 & Montes 2 & Montes 3 \\
\hline OFM & 75,20 & 55,77 & 46,62 \\
\hline Topaze & 257,78 & 62,37 & 51,28 \\
\hline ValNav & 61,73 & 72,40 & 60,71 \\
\hline Forecast & 73,85 & 51,23 & 44,36 \\
\hline Serie de tiempo & 25,21 & 22,86 & 18,15 \\
\hline
\end{tabular}

A pesar de que el comportamiento del modelo de serie de tiempo, se aproxima a una línea de tendencia después de pocas observaciones, se aprecia un menor error, comparado con los resultados de los diferentes programas. 


\section{CONCLUSIONES}

A partir del análisis de los datos de producción se observa que el ajuste exponencial no siempre es el que permite obtener la mejor descripción del comportamiento de los datos.

A pesar de que los pronósticos de producción generados mediante un modelo de serie de tiempo reducen su efectividad en el largo plazo, continúa siendo una herramienta con mayor precisión que las metodologías tradicionales.

Es necesario definir un algoritmo de cálculo para cada pozo, pues la generalización de las técnicas de análisis histórico y predicción conlleva a la ocurrencia de errores significativos.

\section{REFERENCIAS}

1. ADEREMI, Samuel, et al. Effective Use of Production Surveillance Tool in Forecasting Future Production. En Nigeria Annual International Conference and Exhibition. Society of Petroleum Engineers, 2008.

2. ARPS, Jan J., et al. Analysis of decline curves. Transactions of the AIME, 1945, vol. 160, no 01, p. 228-247.

3. CAMPBELL JR, J. M., et al. Forecasting: Art or Science. En SPE Annual Technical Conference and Exhibition. Society of Petroleum Engineers, 1989.

4. FETKOVICH, Michael J., et al. Decline curve analysis using type curves. Journal of Petroleum Technology, 1980, vol. 32, no 06, p. 1,065-1,077.
5. FETKOVICH, M. J., et al. Useful concepts for decline curve forecasting, reserve estimation, and analysis. SPE Reservoir Engineering, 1996, vol. 11, no 01, p. $13-22$.

6. GARCÍA, Á. M. Análisis de series de tiempo. Bogotá: Universidad Javeriana, 2010.

7. GUERRERO, José Felipe Jiménez; FERNÁNDEZ, Raquel Sánchez; ABAD, Juan Carlos Gázquez. La capacidad predictiva en los métodos Box-Jenkins y Holt-Winters: una aplicación al sector turístico. Revista Europea de Dirección y Economía de la Empresa, 2006, vol. 15, no 3, p. 185-198.

8. MANNON, Robert W., et al. Some Aspects Of Production Forecasting. En SPE Rocky Mountain Regional Meeting. Society of Petroleum Engineers, 1964.

9. PAlaciO, J. C.; Blasingame, T. A. Decline curve analysis using type curves - analysis of gas well production data. paper SPE, 1993, vol. 25909, p. 12-14.

10. POSTON, Steven W.; POE, Bobby D. Analysis of production decline curves. Richardson, TX: Society of Petroleum Engineers, 2008.

11. SCHWARZ, Gideon, et al. Estimating the dimension of a model. The annals of statistics, 1978, vol. 6, no 2, p. 461-464.

12. STERNE, Jonathan AC; SMITH, George Davey. Sifting the evidence-what's wrong with significance tests? Physical Therapy, 2001, vol. 81, no 8 , p. 1464-1469.

Recepción: 23 de febrero de 2016

Aceptación: 4 de marzo de 2016 